\title{
Thermodynamic, Electromagnetic, and Lattice Properties of Antiperovskite $\mathrm{Mn}_{3} \mathrm{SbN}$
}

\author{
Ying Sun, ${ }^{1}$ Yan-Feng Guo, ${ }^{2}$ Yoshihiro Tsujimoto, ${ }^{3}$ Xia Wang, ${ }^{2}$ Jun Li, ${ }^{2}$ Clastin I. Sathish, ${ }^{2,4}$ \\ Cong Wang, and Kazunari Yamaura ${ }^{2,4}$ \\ ${ }^{1}$ International Center for Materials Nanoarchitectonics (WPI-MANA), National Institute for Materials Science, 1-1 Namiki, Ibaraki, \\ Tsukuba 305-0044, Japan \\ ${ }^{2}$ Superconducting Properties Unit, National Institute for Materials Science, 1-1 Namiki, Ibaraki, Tsukuba 305-0044, Japan \\ ${ }^{3}$ Materials Processing Unit, National Institute for Materials Science, 1-1 Namiki, Ibaraki, Tsukuba 305-0044, Japan \\ ${ }^{4}$ Graduate School of Chemical Sciences and Engineering, Hokkaido University, Hokkaido, Sapporo 060-0810, Japan \\ ${ }^{5}$ Department of Physics, Center for Condensed Matter and Materials Physics, Beihang University, Beijing, Haidian 100191, China
}

Correspondence should be addressed to Ying Sun; sun.ying@nims.go.jp

Received 1 October 2012; Revised 4 December 2012; Accepted 5 December 2012

Academic Editor: Y. Sun

Copyright (c) 2013 Ying Sun et al. This is an open access article distributed under the Creative Commons Attribution License, which permits unrestricted use, distribution, and reproduction in any medium, provided the original work is properly cited.

The physical properties of polycrystalline $\mathrm{Mn}_{3} \mathrm{SbN}$ were investigated using measurements of the magnetic, calorimetric, and electronic transport properties. At room temperature, the phase crystallizes in a tetragonal structure with $P 4 / \mathrm{mmm}$ symmetry. A remarkably sharp peak in the heat capacity versus temperature curve was found near $353 \mathrm{~K}$. The peak reaches $723 \mathrm{~J} \mathrm{~mol}^{-1} \mathrm{~K}^{-1}$ at its highest, which corresponds to a transition entropy of $10.2 \mathrm{~J} \mathrm{~mol}^{-1} \mathrm{~K}^{-1}$. The majority of the large entropy change appears to be due to lattice distortion from the high-temperature cubic structure to the room-temperature tetragonal structure and the accompanying Ferrimagnetic transition.

\section{Introduction}

Antiperovskite compounds with the formula $\mathrm{Mn}_{3} \mathrm{XN}$ or $\mathrm{Mn}_{3} \mathrm{XC}(\mathrm{X}=\mathrm{Cu}, \mathrm{Zn}, \mathrm{Ga}, \mathrm{Cu}, \mathrm{In}$, or $\mathrm{Sn})$ were discovered in the middle of the last century [1]. Recently, interest in these materials has intensively renewed owing to discoveries of new, potentially useful properties [2-4] such as the giant magnetoresistance of $\mathrm{Mn}_{3} \mathrm{GaC}$ [5], negative thermal expansion (NTE) of $\mathrm{Mn}_{3} \mathrm{Cu}(\mathrm{Ge}) \mathrm{N}$ [6] and $\mathrm{Mn}_{3} \mathrm{Zn}(\mathrm{Ge}) \mathrm{N}$ [7], magnetostriction of $\mathrm{Mn}_{3} \mathrm{CuN}$ [8] and $\mathrm{Mn}_{3} \mathrm{SbN}$ [9], and nearzero temperature coefficient of the resistivity of $\mathrm{Mn}_{3} \mathrm{CuN}$ [10] and $\mathrm{Mn}_{3} \mathrm{NiN}$ [11]. Specifically, Takenaka and Takagi found that $\mathrm{Ge}$-doped $\mathrm{Mn}_{3} \mathrm{CuN}$ compound has a large NTE $\left(\right.$ NTE parameter $\left.=-25 \times 10^{-6} \mathrm{~K}^{-1}\right)$ [12]; using neutron diffraction, the broad NTE was determined to be associated with the local $\mathrm{T}_{4}$ structure [6]. Asano et al. discovered large magnetostriction in tetragonal $\mathrm{Mn}_{3} \mathrm{CuN}$; it expands $0.2 \%$ and shrinks $0.1 \%$ in the directions parallel and perpendicular to an external $90 \mathrm{kOe}$ magnetic field, respectively [8]. In previous studies, we found a peculiar phase separation and accompanying anomaly in the electronic transport properties of $\mathrm{Mn}_{3} \mathrm{ZnN}[13,14]$, while further study indicated that the thermal expansion properties of $\mathrm{Mn}_{3} \mathrm{ZnN}$ can be controlled by introducing $\mathrm{Zn}$ vacancies [15]. In addition, Song et al. observed a canonical spin-glass state in $\mathrm{Mn}_{3} \mathrm{GaN}$ below the spin-freezing temperature of $133 \mathrm{~K}$ [16]. Lukashev et al. systematically studied the spin density of the spin-frustrated state of a Mn-based antiperovskite under mechanical stress [17].

The above-mentioned properties enable a variety of potential applications for this type of material. Although the prospective industrial markets are expected to be large and much effort has already been devoted to studying their structural, electromagnetic, and transport properties, further investigations on antiperovskite materials are still required. In this study, the thermodynamic, electromagnetic, and electronic transport properties of $\mathrm{Mn}_{3} \mathrm{SbN}$ are investigated. In particular, we focused on the notable transition entropy that 


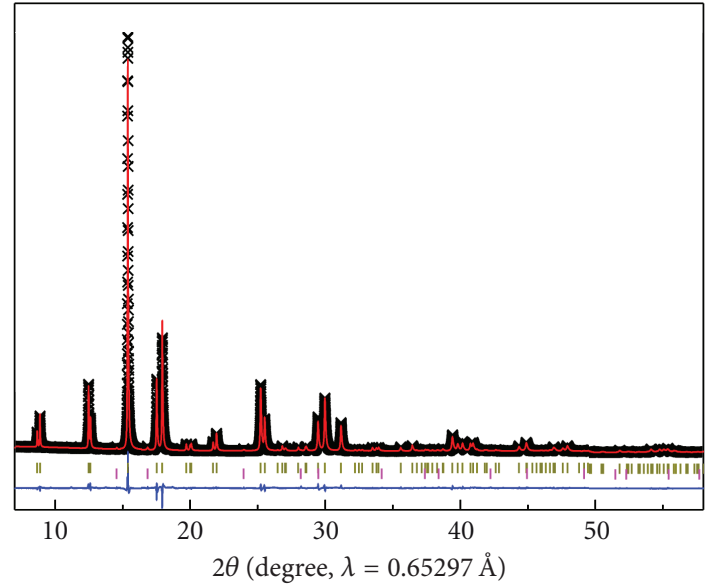

FIGURE 1: Rietveld refinement of the synchrotron XRD pattern of $\mathrm{Mn}_{3} \mathrm{SbN}$. The crosses and solid lines represent the observed and calculated profiles, respectively, and the difference between the curves is shown at the bottom. Bragg positions are marked by small ticks (upper: $\mathrm{Mn} 3 \mathrm{ZnN}$, lower: $\mathrm{MnO}$ ).

accompanies the magnetic and crystal structure transition above room temperature.

\section{Experimental Details}

A polycrystalline $\mathrm{Mn}_{3} \mathrm{SbN}$ sample was prepared via the solid-state reaction of fine powders of $\mathrm{Sb}$ (99.99\%, Rare Metallic Co.) and $\mathrm{Mn}_{2} \mathrm{~N}$, which was synthesized by firing $\mathrm{Mn}$ powder (99.99\%, Sigma Aldrich Co.) in nitrogen at $800^{\circ} \mathrm{C}$ for $60 \mathrm{~h}$. Stoichiometric amounts of the starting materials were thoroughly mixed, and the mixture was pressed into a pellet. The pellet was sealed in an evacuated quartz tube, heated in a box furnace at $800^{\circ} \mathrm{C}$ for three days, and then slowly cooled to room temperature in the furnace.

The crystal structure of $\mathrm{Mn}_{3} \mathrm{SbN}$ was analyzed by synchrotron X-ray diffraction (SXRD) using a large DebyeScherrer camera at the BL15XU NIMS beam line of the SPring-8 facility in Hyogo, Japan. The SXRD data were collected for $2 \theta$ ranging from $2^{\circ}$ to $60^{\circ}$ at intervals of $0.003^{\circ}$. The incident beam was monochromatized at $\lambda=0.65297 \AA$. The evolution of the $\mathrm{Mn}_{3} \mathrm{SbN}$ crystal structure with temperature was also determined via the measurement of the SXRD patterns.

The temperature dependence of magnetization was measured between 2 and $400 \mathrm{~K}$ with applied magnetic fields of 0.1 and $5 \mathrm{kOe}$ using a Magnetic Property Measurements System (Quantum Design). The measurements were conducted on loosely gathered powder under both zero-field cooling (ZFC) and field cooling (FC) conditions. The isothermal magnetization curve was recorded at $10 \mathrm{~K}$ between -50 and $50 \mathrm{kOe}$.

Specific heat $\left(C_{p}\right)$ values were measured between 2 and $400 \mathrm{~K}$ with cooling using a Physical Properties Measurement System (Quantum Design). The sample was fixed on a stage using a small amount of grease; the heat capacity of the grease was measured first and subtracted from the total $C_{p}$.

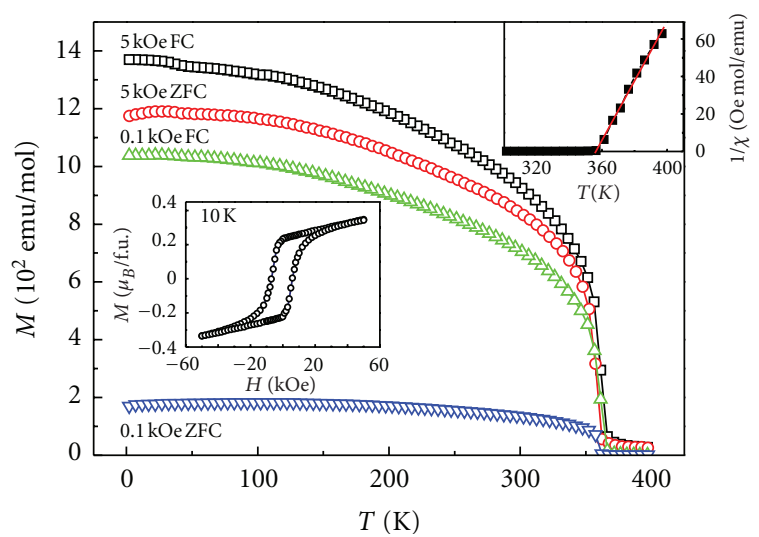

FIGURE 2: Temperature dependence of magnetization in magnetic fields of 0.1 and $5 \mathrm{kOe}$. The left and right insets show the isothermal magnetization curve at $10 \mathrm{~K}$ and $\chi^{-1}$ versus $T$ plot of the FC data at $0.1 \mathrm{kOe}$, respectively.

The electrical resistivity $(\rho)$ was measured between 2 and $400 \mathrm{~K}$ with cooling and heating using a conventional fourprobe techniques with the same apparatus. The AC gauge current and frequency were $10 \mathrm{~mA}$ and $30 \mathrm{~Hz}$, respectively. The electrical contacts were prepared on the surface of a barshaped piece of the pellet using silver paste and $\mathrm{Pt}$ wires.

\section{Results and Discussion}

As shown in Figure 1, the synchrotron XRD pattern at room temperature fit well with a model pattern of the proposed structure (space group: $P 4 / \mathrm{mmm}$ ). The structural parameters of $\mathrm{Mn}_{3} \mathrm{SbN}$ were refined by the Rietveld method using the RIETAN-FP program [18]. The occupancy factors of Sb, $\mathrm{N}, \mathrm{Mn} 1$, and Mn2 were refined to be 1 (fixed), 1 (fixed), 0.97(1), and 0.99(1), respectively, while the isotropic atomic displacement parameters were $0.42(1), 0.84(5), 0.86(1)$, and $0.78(1) \AA^{2}$, respectively. The lattice constants were calculated to be $a=b=4.17994(4) \AA$ and $c=4.27718(5) \AA$. The final $R_{\mathrm{wp}}$ and $R_{p}$ reliability indexes were below $5.56 \%$ and $4.09 \%$, respectively. The analysis revealed 1.91 mass $\% \mathrm{MnO}$ in the sample as an impurity; as shown later, the magnetic, $C_{p}$, and $\rho$ measurements suggest that the impurity does not significantly impact the measurements of $\mathrm{Mn}_{3} \mathrm{SbN}$ in this study.

Figure 2 displays the temperature dependence of magnetization of polycrystalline $\mathrm{Mn}_{3} \mathrm{SbN}$. The magnetization steeply increases upon cooling to around $353 \mathrm{~K}$, which suggests the establishment of long-range magnetic order at the magnetic transition temperature $\left(T_{c}\right)$. In addition, a small hysteresis can be observed between the heating and cooling process, implying the first-order character of the magnetic transition. The remarkable bifurcation between the ZFC and FC curves may originate from the spontaneous alignment of random magnetic $\mathrm{Mn}$ moments in domain boundaries. It is worth noting that the hysteresis is less significant at a higher magnetic field of $5 \mathrm{kOe}$, which supports the domain picture. 


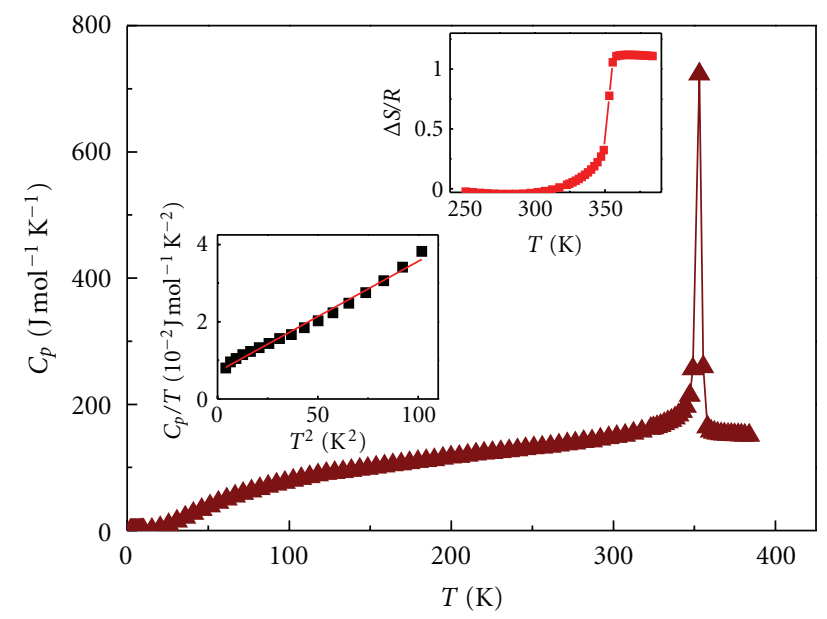

Figure 3: Temperature dependence of the $C_{p}$ of $\mathrm{Mn}_{3} \mathrm{SbN}$. The left and right insets show a linear fit to the $C_{p} / T$ versus $T^{2}$ curve and an estimation of $\Delta S$, respectively.

To further study the magnetic properties, we applied the Curie-Weiss law to the paramagnetic portion. As shown in the right inset of Figure 2, the $\chi^{-1}-T$ plot is well represented by the Curie-Weiss law, that is, the spin-only expression for magnetic susceptibility: $\chi(T)=C /\left(T-\Theta_{W}\right)$, where $C$ is the Curie constant and $\Theta_{W}$ is the Weiss temperature. The value of $\Theta_{W}$ was determined to be $354 \mathrm{~K}$, which suggests that ferromagnetic correlation is dominant in the spin system. The effective Bohr magneton $\left(\mu_{\text {eff }}\right)$ was estimated to be $1.28 \mu_{B} / \mathrm{Mn}$ from $\mu_{\text {eff }}=2.83(\mathrm{C} / \eta)^{0.5} \mu_{B}$, where $\eta$ is the number of magnetic atoms in the molecular formula $(\eta=3$ in the present case). The value of $\mu_{\text {eff }}$ is much lower than that of other antiperovskite manganese nitrides (e.g., $2.87 \mu_{B}$ for $\mathrm{Mn}_{3} \mathrm{ZnN}$ [14]) and even lower than the expected moment for localized $S=1 / 2$ spins, suggesting an itinerant character of the $3 \mathrm{~d}$ electrons in $\mathrm{Mn}_{3} \mathrm{SbN}$.

From the isothermal magnetization curve (see the inset of Figure 2), it was found that the magnetization at $50 \mathrm{kOe}$ is $\sim 0.35 \mu_{B} / \mathrm{Mn}$, which is too small to be caused by full ferromagnetic order. The gap suggests that the spins of the Mn atoms are possibly Ferrimagnetically ordered. This Ferrimagnetic interaction is also suggested by the magnetization characteristics above $10 \mathrm{kOe}$, that is, the magnetization continuously increases with increasing magnetic field without approaching saturation. The Ferrimagnetic order of a related Mn-based antiperovskite compound was explained by a $\Gamma^{4 g}$ spin structure, where two of the three Mn magnetic moments are antiferromagnetically coupled and the third exhibits FM behavior [19]. It is possible that a similar magnetic structure is established in $\mathrm{Mn}_{3} \mathrm{SbN}$ below $353 \mathrm{~K}$.

To further characterize the magnetic transition, the specific heat was measured from 400 to $2 \mathrm{~K}$. As shown in Figure 3 , the temperature dependence of $C_{p}$ features a sharp and narrow peak around $T_{c}\left(\Delta C_{p} / R=87\right.$ and $\Delta T=3 \mathrm{~K}$, where $R$ is the ideal gas constant). This is indicative of a first-orderlike transition, as discussed in [20].

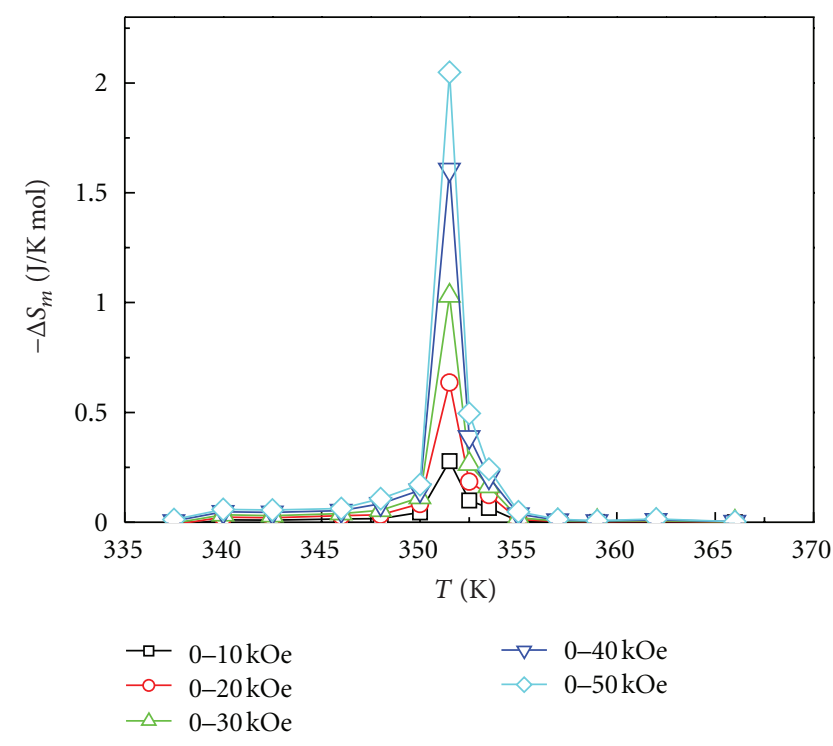

FIGURE 4: Temperature dependence of the magnetic entropy change when the magnetic field changes from 0 to 10, 20, 30, 40, and $50 \mathrm{kOe}$, respectively.

An estimation of entropy change is essential to understanding the nature of the transition of $\mathrm{Mn}_{3} \mathrm{SbN}$. The peak was roughly separated from the baseline using a polynomial function. Analysis indicates that the total transition entropy $(\Delta S)$ is $\sim 1.23 R(10.2 \mathrm{~J} / \mathrm{mol} \mathrm{K})$. Since the total entropy change comprises all contributions, including the lattice, electronic, and magnetic changes [20], we evaluated each contribution independently.

For the present compound, the abrupt change of the magnetization at $T_{c}$ may induce a large $\partial M / \partial T$; therefore, a large magnetic entropy change $\left(\Delta S_{m}\right)$ is expected. A series of magnetization curves with small temperature steps were measured; the data allow for a rough estimation of the magnetic entropy change via the thermodynamic Maxwell relation, as follows [21]:

$$
\left(\frac{\partial S(T, H)}{\partial H}\right)_{T}=-\left(\frac{\partial M(T, H)}{\partial T}\right)_{H} .
$$

The magnetic entropy change, $\Delta S_{m}(T, H)$, can be calculated by

$\Delta S_{m}(T, H)=S_{m}(T, H)-S_{m}(T, 0)=\int_{0}^{H}\left(\frac{\partial M(T, H)}{\partial T}\right)_{H} d H$.

The temperature dependence of $\Delta S_{m}$ calculated from (2) with fields of 10, 20, 30, 40, and $50 \mathrm{kOe}$ is shown in Figure 4. The $\Delta S_{m}$ is maximized around $T_{c}$, and the maximum is estimated to be $\sim 2.1 \mathrm{~J} \mathrm{~mol}^{-1} \mathrm{~K}^{-1}$, which implies that the lattice and electronic changes provide a fairly large contribution to the total entropy change.

To investigate the electronic contribution (i.e., the Sommerfeld coefficient or $\gamma$ ), the $C_{p} / T$ versus $T^{2}$ plot below $10 \mathrm{~K}$ was analyzed by applying the approximate Debye model, as 


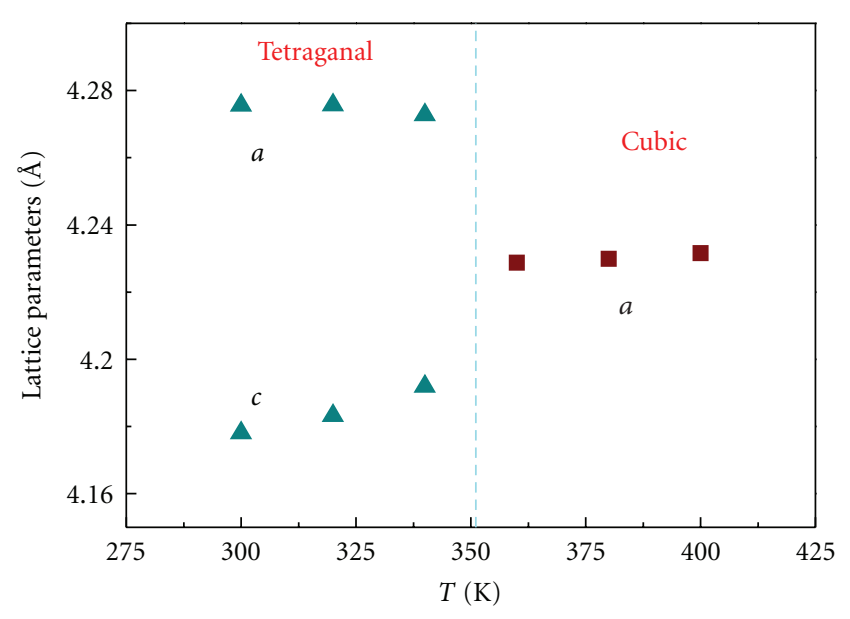

(a)

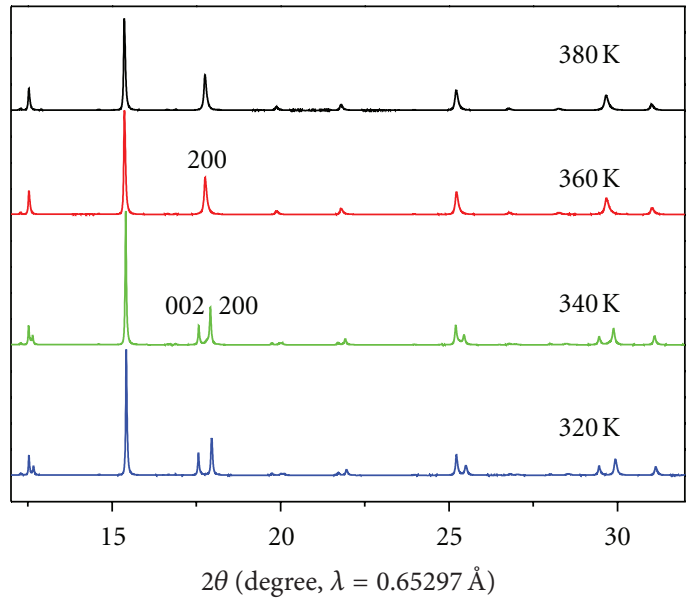

(b)

FIGURE 5: Variation in (a) lattice parameters and (b) synchrotron XRD patterns with temperature for $\mathrm{Mn}_{3} \mathrm{SbN}_{\text {. }}$

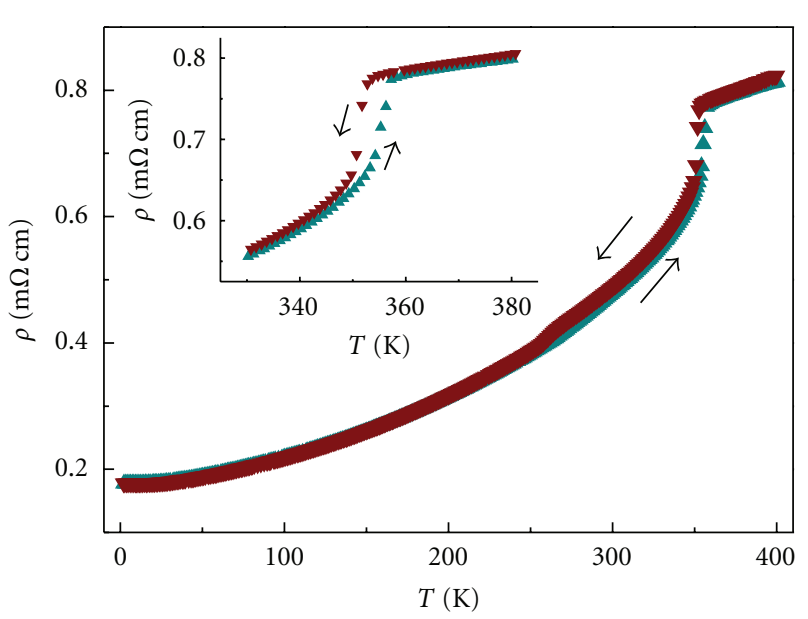

FigURE 6: Variation of $\rho$ with temperature for $\mathrm{Mn}_{3} \mathrm{SbN}$ upon cooling and warming. The inset shows an enlarged view of the $\rho$ variation around the magnetic transition.

follows: $C(T) / T=\gamma+2.4 \pi^{4} n N_{0} k_{B}\left(1 / \Theta_{D}{ }^{3}\right) T^{2}\left(T \ll \Theta_{D}\right)$, where $n$ denotes the number of atoms per formula unit, $k_{B}$ is the Boltzmann constant, $N_{0}$ is the Avogadro constant, and $\Theta_{D}$ is the Debye temperature. Fitting to the linear part of the $C_{p} / T$ versus $T^{2}$ plot using the least-squares method yielded $\gamma$ and $\Theta_{D}$ values of $\sim 7.03(1) \mathrm{mJ} \mathrm{mol}^{-1} \mathrm{~K}^{-2}$ and $326(2) \mathrm{K}$, respectively. Compared with the parameters determined for other antiperovskite nitrides, $\mathrm{Mn}_{3} \mathrm{SbN}$ has a much lower $\gamma$, which indicates that the electronic correlation is somewhat weakened [20]. Thus, the electronic contribution might not be a dominant contributor to the total transition entropy.

In addition to the magnetic and electronic contributions, a possible lattice change may need to be investigated to analyze the total transition entropy. The variation of the synchrotron XRD pattern with temperature was measured. As shown in Figure 5(b). It can be seen that some typical reflections disappear with temperature, for example, the two reflections (002) and (200) for the $P 4 / \mathrm{mmm}$ lattice merge to one reflection. By the Rietveld analysis of the synchrotron XRD patterns, the structural change from tetragonal to cubic was defined, and the lattice constants were determined as a function of temperature, as shown in Figure 5(a). It is obvious that lattice parameter $c$ increases slightly with increasing temperature, whereas a gradually decreases. When the temperature crosses $T_{c}$, the tetragonal structure completely transforms to an unidentified cubic structure. Hence, the lattice distortion must contribute to the total entropy change.

According to the thermodynamic relation, the magnetization $(M)$ is equal to the first derivative of the magnetic free energy by the magnetic field, that is, $d f(M) / d H$ [22]. Therefore, the sharp transition indicates that the energy barrier in the free energy that separates the paramagnetic and ferromagnetic states is large. Accordingly, $T_{c}$ and the energy barrier height probably correlate with the electronic density of states, which exhibits a sharp peak near the Fermi level [23]; therefore, the large entropy change is possibly related to the reconstruction of the electronic structure, which could induce the magnetic and structural transition. Since such an electronic reconstruction is often sharply reflected in a $\rho-T$ curve, the electronic transport properties of $\mathrm{Mn}_{3} \mathrm{SbN}$ were carefully measured (shown in Figure 6). It is evident that an abnormal drop appears at $T_{c}$ in the $\rho$-T curve, which is indicative of an electronic structure reconstruction. In addition, as shown in the inset of Figure 6, a small hysteresis was observed between the warming and cooling curves; this is in agreement with a first-order transition.

\section{Conclusions}

In conclusion, the thermodynamic, electromagnetic, and transport properties of antiperovskite $\mathrm{Mn}_{3} \mathrm{SbN}$ were studied. The phase crystallizes in a tetragonal structure with $a=b$ $=4.17994(4) \AA$ and $c=4.27718(5) \AA$ at room temperature. The $C_{p}$ measurements revealed a sharp endothermic peak in the $C_{p}-T$ curve at $353 \mathrm{~K}$, which corresponds to a large 
entropy change $\left(\sim 10.2 \mathrm{~J} \mathrm{~mol}^{-1} \mathrm{~K}^{-1}\right)$. The present study clearly indicates that the entropy change is accompanied with a Ferrimagnetic transition and lattice distortion as well as a possible electronic structure reconstruction.

\section{Conflict of Interests}

The authors declare that they have no conflict of interests.

\section{Acknowledgments}

The authors would like to thank the staff members at BL15XU, the National Institute for Materials Science (NIMS), and SPring-8 for their help in the use of the beamline. The SXRD measurements were performed with the approval of the NIMS Beamline Station (Proposal no. 2011A4502). This study was supported in part by the World Premier International Research Center Initiative of the Ministry of Education, Culture, Sports, Science, and Technology (MEXT); a Grant-in-Aid for Scientific Research grant (no. 22246083) from the Japan Society for the Promotion of Science (JSPS); the Funding Program for World-Leading Innovative R\&D on Science and Technology (FIRST Program) of the JSPS; the Advanced Low Carbon Technology Research and Development Program (ALCA) of the Japan Science and Technology Agency (JST).

\section{References}

[1] G. W. Wiener and J. A. Berger, "Structure and magnetic properties of some transition metal nitrides," Transactions of the American Institute of Mining, Metallurgical, and Petroleum Engineers, vol. 203, pp. 360-368, 1955.

[2] Y. Sun, Y. C. Wen, L. H. Chu, M. Nie, and C. Wang, "Abnormal thermal expansion in anti-perovskite manganese nitride," Journal of the Chinese Ceramic Society, vol. 37, no. 5, pp. 73-77, 2009.

[3] B. S. Wang, P. Tong, Y. P. Sun et al., "Large magnetic entropy change near room temperature in antiperovskite $\mathrm{SnCMn}_{3}$," Environment People Law, vol. 85, no. 4, Article ID 47004, 2009.

[4] R. Huang, L. Li, F. Cai, X. Xu, and L. Qian, "Low-temperature negative thermal expansion of the antiperovskite manganese nitride $\mathrm{Mn}_{3} \mathrm{CuN}$ codoped with $\mathrm{Ge}$ and Si," Applied Physics Letters, vol. 93, no. 8, Article ID 081902, 3 pages, 2008.

[5] K. Kamishima, T. Goto, H. Nakagawa et al., "Giant magnetoresistance in the intermetallic compound $\mathrm{Mn}_{3} \mathrm{GaC}$," Physical Review B, vol. 63, no. 2, Article ID 024426, 3 pages, 2001.

[6] S. Iikubo, K. Kodama, K. Takenaka, H. Takagi, M. Takigawa, and S. Shamoto, "Local lattice distortion in the giant negative thermal expansion material $\mathrm{Mn}_{3} \mathrm{Cu}_{1-x} \mathrm{Ge}_{x} \mathrm{~N}$," Physical Review Letters, vol. 101, no. 20, Article ID 205901, 4 pages, 2008.

[7] Y. Sun, C. Wang, Y. C. Wen, K. G. Zhu, and J. T. Zhao, "Lattice contraction and magnetic and electronic transport properties of $\mathrm{Mn}_{3} \mathrm{Zn}_{1-x} \mathrm{Ge}_{x} \mathrm{~N}$," Applied Physics Letters, vol. 91, Article ID 231913, 3 pages, 2007.

[8] K. Asano, K. Koyama, and K. Takenaka, "Magnetostriction in $\mathrm{Mn}_{3} \mathrm{CuN}$," Applied Physics Letters, vol. 92, no. 16, Article ID 161909, 3 pages, 2008.
[9] T. Shimizu, T. Shibayama, K. Asano, and K. Takenaka, "Giant magnetostriction in tetragonally distorted antiperovskite manganese nitrides," Journal of Applied Physics, vol. 111, Article ID 07A903, 3 pages, 2012.

[10] E. O. Chi, W. S. Kim, and N. H. Hur, "Nearly zero temperature coefficient of resistivity in antiperovskite compound $\mathrm{CuNMn}_{3}$," Solid State Communications, vol. 120, no. 7-8, pp. 307-310, 2001.

[11] Y. Sun, C. Wang, L. Chu, Y. Wen, M. Nie, and F. Liu, "Low temperature coefficient of resistivity induced by magnetic transition and lattice contraction in $\mathrm{Mn}_{3} \mathrm{NiN}$ compound," Scripta Materialia, vol. 62, no. 9, pp. 686-689, 2010.

[12] K. Takenaka and H. Takagi, "Giant negative thermal expansion in Ge-doped anti-perovskite manganese nitrides," Applied Physics Letters, vol. 87, no. 26, Article ID 261902, 3 pages, 2005.

[13] Y. Sun, C. Wang, Q. Z. Huang et al., "Neutron diffraction study of unusual phase separation in the antiperovskite nitride $\mathrm{Mn}_{3} \mathrm{ZnN}$," Inorganic Chemistry, vol. 51, no. 13, pp. 7232-7236, 2012.

[14] Y. S. Sun, Y. F. Guo, X. X. Wang et al., "Resistive switching phenomenon driven by antiferromagnetic phase separation in an antiperovskite nitride $\mathrm{Mn}_{3} \mathrm{ZnN}$," Applied Physics Letters, vol. 100, Article ID 161907, 4 pages, 2012.

[15] C. Wang, L. H. Chu, Q. R. Yao et al., "Tuning the range, magnitude, and sign of the thermal expansion in intermetallic $\mathrm{Mn}_{3}(\mathrm{Zn}, \mathrm{M})_{x} \mathrm{~N}(\mathrm{M}=\mathrm{Ag}, \mathrm{Ge})$, , Physical Review B, vol. 85, Article ID 220103, 5 pages, 2012.

[16] B. Song, J. Jian, H. Bao et al., "Observation of spin-glass behavior in antiperovskite $\mathrm{Mn}_{3} \mathrm{GaN}$," Applied Physics Letters, vol. 92, no. 19, Article ID 192511, 3 pages, 2008.

[17] P. Lukashev, R. F. Sabirianov, and K. Belashchenko, "Theory of the piezomagnetic effect in Mn-based antiperovskites," Physical Review B, vol. 78, no. 18, Article ID 184414, 5 pages, 2008.

[18] F. Izumi and K. Momma, "Three-dimensional visualization in powder diffraction," Solid State Phenomena, vol. 130, pp. 15-20, 2007.

[19] D. Fruchart and E. F. Bertaut, "Magnetic studies of the metallic perovskite-type compounds of manganese," Journal of the Physical Society of Japan, vol. 44, no. 3, pp. 781-791, 1978.

[20] J. García, J. Bartolomé, D. González, R. Navarro, and D. Fruchart, "Thermophysical properties of the intermetallic $\mathrm{Mn}_{3} \mathrm{MN}$ perovskites II. Heat capacity of manganese zinc nitride: $\mathrm{Mn}_{3} \mathrm{ZnN}$ and manganese gallium nitride: $\mathrm{Mn}_{3} \mathrm{GaN}$," The Journal of Chemical Thermodynamics, vol. 15, no. 11, pp. 1041-1057, 1983.

[21] M. H. Phan and S. C. Yu, "Review of the magnetocaloric effect in manganite materials," Journal of Magnetism and Magnetic Materials, vol. 308, no. 2, pp. 325-340, 2007.

[22] A. Fujita, S. Fujieda, Y. Hasegawa, and K. Fukamichi, "Itinerantelectron metamagnetic transition and large magnetocaloric effects in $\mathrm{La}\left(\mathrm{Fe}_{x} \mathrm{Si}_{1-x}\right)_{13}$ compounds and their hydrides," Physical Review B, vol. 67, no. 10, Article ID 104416, 2003.

[23] J. P. Jardin and J. Labbe, "Phase transitions and band structure in metallic perovskites (carbides and nitrides)," Journal of Solid State Chemistry, vol. 46, pp. 275-293, 1983. 

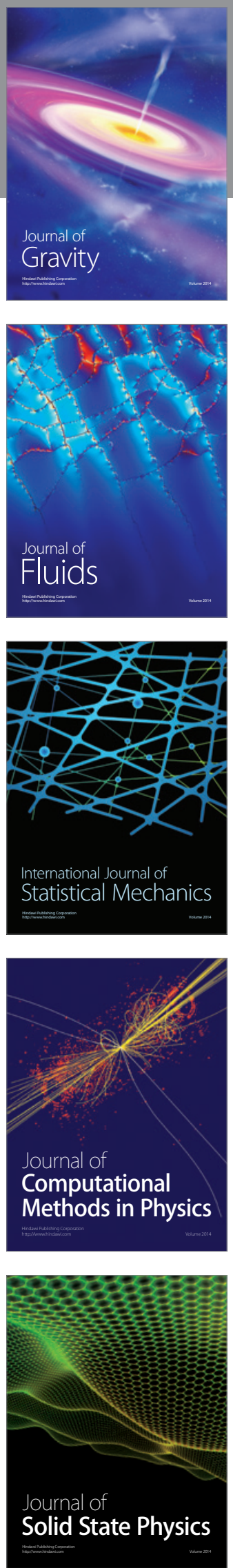

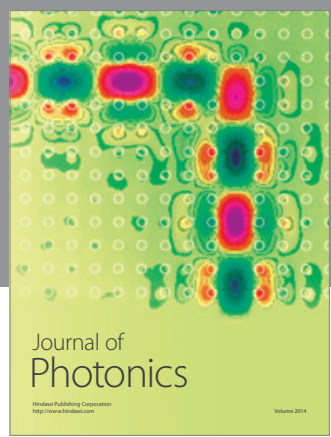

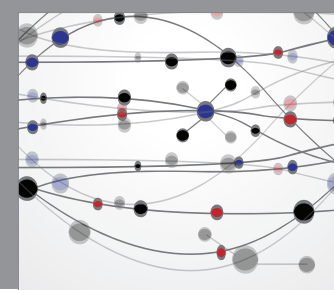

The Scientific World Journal

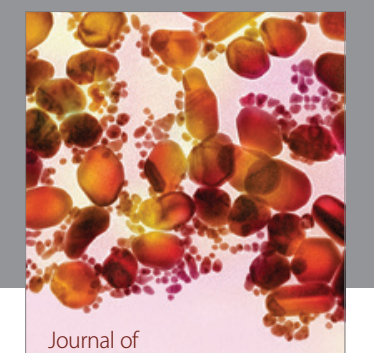

Soft Matter
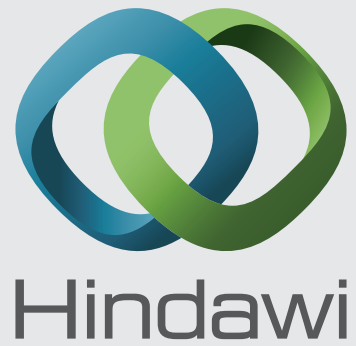

Submit your manuscripts at

http://www.hindawi.com
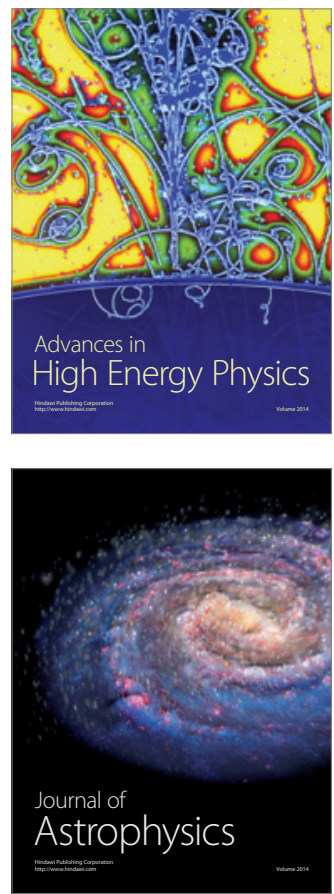
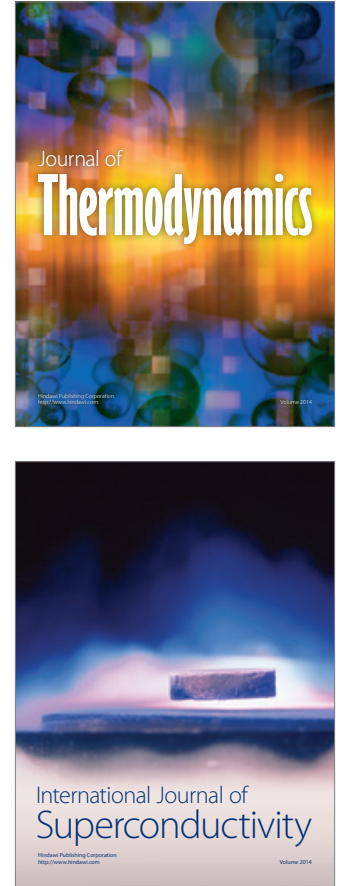
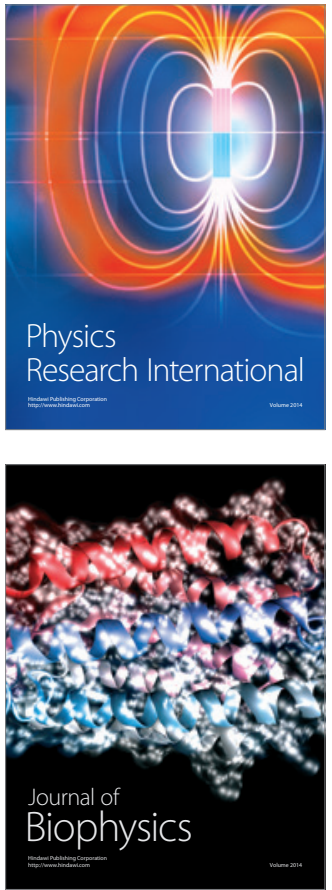
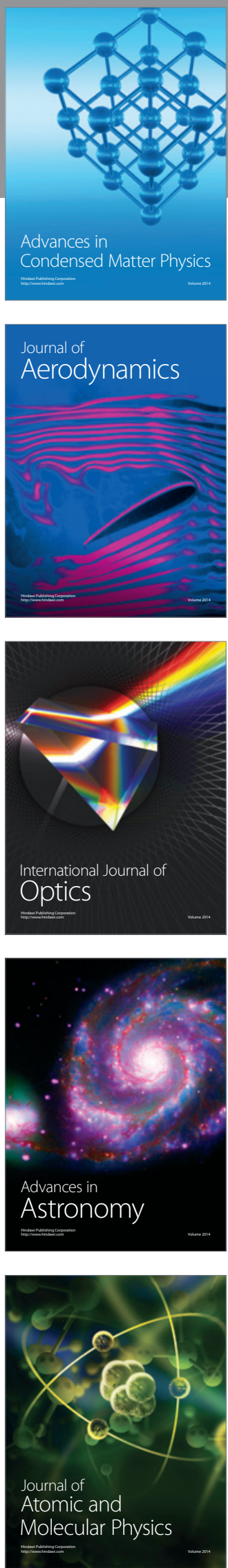